\title{
Verbal Dyspraxia and Galactosemia
}

\author{
AMY LEIGH WEBB, RANI H. SINGH, MARY JANE KENNEDY, AND LOUIS J. ELSAS \\ Emory University Graduate School of Arts and Sciences, Department of Biological and Biomedical \\ Sciences, Division of Nutrition and Health Sciences [A.L.W], School of Medicine, Department of \\ Pediatrics, Division of Medical Genetics [R.H.S., M.J.K.], Atlanta, Georgia 30322, U.S.A., and University \\ of Miami School of Medicine, Center for Medical Genetics, Miami, Florida 33101, U.S.A. [L.J.E.]
}

\begin{tabular}{|c|c|}
\hline \multicolumn{2}{|c|}{ ABSTRACT } \\
\hline $\begin{array}{l}\text { Classical galactosemia is an autosomal recessive disorder } \\
\text { resulting from deficient galactose-1-phosphateuridyl transferase } \\
\text { (GALT) activity. Verbal dyspraxia is an unusual outcome in } \\
\text { galactosemia. Here we validated a simplified breath test of total } \\
\text { body galactose oxidation against genotype and evaluated five } \\
\text { potential biochemical risk indicators for verbal dyspraxia in } \\
\text { galactosemia: cumulative percentage dose (CUMPCD) of }{ }^{13} \mathrm{CO}_{2} \\
\text { in breath, mean erythrocyte galactose-1-phosphate, highest } \\
\text { erythrocyte galactose-1-phosphate, mean urinary galactitol, and } \\
\text { erythrocyte GALT activity. Thirteen controls and } 42 \text { patients } \\
\text { with galactosemia took a }{ }^{13} \mathrm{C} \text {-galactose bolus, and the } \\
\text { (CUMPCD) of }{ }^{13} \mathrm{CO}{ }_{2} \text { in expired air was determined. Patients } \\
\text { with }<5 \% \mathrm{CUMPCD} \text { had mutant alleles that severely impaired } \\
\text { human GALT enzyme catalysis. Patients with } \geq 5 \% \mathrm{CUMPCD} \\
\text { had milder mutant human GALT alleles. Twenty-four patients } \\
\text { consented to formal speech evaluation; } 15(63 \%) \text { had verbal } \\
\text { dyspraxia. Dyspraxic patients had significantly lower CUMPCD } \\
\text { values ( } 2.84 \pm 5.76 \% \text { versus } 11.51 \pm 7.67 \% ; p<0.008) \text { and } \\
\text { significantly higher mean erythrocyte galactose-1-phosphate }\end{array}$ & $\begin{array}{l}(3.38 \pm 0.922 \mathrm{mg} / \mathrm{dL} \text { versus } 1.92 \pm 1.28 \mathrm{mg} / \mathrm{dL} ; p=0.019) \text { and } \\
\text { mean urinary galactitol concentrations }(192.4 \pm 75.8 \mathrm{mmol} / \mathrm{mol} \\
\text { creatinine versus } 122.0 \pm 56.4 ; p=0.048) \text { than patients with } \\
\text { normal speech. CUMPCD values }<5 \% \text {, mean erythrocyte galac- } \\
\text { tose-1-phosphate levels }>2.7 \mathrm{mg} / \mathrm{dL} \text {, and mean urinary galactitol } \\
\text { levels }>135 \mathrm{mmol} / \mathrm{mol} \text { creatinine were associated with dys- } \\
\text { praxic outcome with odds ratios of } 21,13 \text {, and } 5 \text {, respectively. } \\
\text { We conclude that total body oxidation of galactose to } \mathrm{CO}_{2} \text { in } \\
\text { expired air reflects genotype and that this breath test is a sensitive } \\
\text { predictor of verbal dyspraxia in patients with galactosemia. } \\
\text { (Pediatr Res 53: 396-402, 2003) } \\
\text { Abbreviations } \\
\text { hGALT, human galactose-1-phosphate-uridyltransferase } \\
\text { hUGP2, human UDP-glucose pyrophosphorylase } \\
\text { UDP-gal, UDP-galactose } \\
\text { UDP-glu, UDP-glucose } \\
\text { OR, odds ratio } \\
\text { CUMPCD, cumulative percentage dose }\end{array}$ \\
\hline
\end{tabular}

Classical galactosemia is an autosomal recessive disorder resulting from deficient human galactose-1-phosphate uridyl transferase (hGALT) $(1,2)$. Currently, $>180$ mutations produce $<1 \%$ of hGALT activity when present as homozygous or compound heterozygous mutant genotypes (3-5). Newborns with galactosemia exhibit elevated concentrations of erythrocyte galactose-1-phosphate and excess galactitol in the urine. If newborn screening, retrieval, diagnosis, and removal of galactose from the diet occur before 5-7 d of life, then the neonatal signs of hepatotoxicity, jaundice, anorexia, and diarrhea are prevented or resolve $(6,7)$. However, infants who have classical galactosemia and are treated continue to have elevated

Received June 19, 2002; accepted November 6, 2002.

Correspondence: Louis J Elsas, M.D., The Dr. John T. Macdonald Foundation Center for Medical Genetics, University of Miami School of Medicine, P.O. Box 016820/(D820), Miami, FL 33101, U.S.A.; e-mail: lelsas@med.miami.edu

This research was supported in part by a grant from the Zoé Rochat Foundation for Research in Galactosemia, from Advanced Breath Diagnostics, LLC, and U.S. Public Health Services Grant MO1-RR00039 to the General Clinical Research Center of Emory University

DOI: 10.1203/01.PDR.0000049666.19532.1B erythrocyte galactose-1-phosphate and urinary galactitol concentrations after initiation of a galactose-restricted diet. These compounds remain elevated throughout the life of the patient; are indicators of the environment (compliance), genotype, epigenetic phenomenon (8-10); and are implicated in the enigmatic outcomes in galactosemia $(7,8,10-13)$.

Roughly half of the children with classical galactosemia manifest verbal dyspraxia $(7,10,14)$. This expressive speech problem results from an impaired ability to program the muscles needed for speech. Children exhibit "chaotic" speech that commonly manifests in poor diadochokinesis, inconsistent errors with vowels and diphthongs, word "re-ordering," and increasing error with increasing response or complexity. Receptive language is usually normal. Reversal of this disorder is most effective when tailored speech therapy is delivered early in speech development $(10,15)$. Therefore, it would be beneficial to predict this outcome early in life and intervene before the child is labeled with a "learning disability." In previous studies, we found that mean erythrocyte galactose-1-phosphate 
levels $>3.28 \mathrm{mg} / \mathrm{dL}$ and homozygosity for the Q188R allele in hGALT increased the probability for a dyspraxic outcome (10).

Because hGALT activity in peripheral erythrocytes may not indicate hGALT activity in other organs, we developed an oral 2 -h breath test that measures the percentage of labeled ${ }^{13} \mathrm{C}$-Dgalactose to ${ }^{13} \mathrm{CO}_{2}$ in expired air (16). We stratified patients with "classic" galactosemia into two categories of impaired total body galactose oxidation, $<5 \%$ and $\geq 5 \%$ cumulative percentage dose (CUMPCD) recovered at $120 \mathrm{~min}$. Some mutant alleles that reduced erythrocyte hGALT catalysis were associated with very low total body oxidation, whereas others had near-normal oxidation of galactose to expired $\mathrm{CO}_{2}$. For example, individuals with an S135L allele had $<1 \%$ of hGALT activity in anucleated red blood cells but oxidized galactose to $\mathrm{CO}_{2}$ at rates similar to normal individuals (17-19). The Q188R and K285N alleles, however, impaired both enzyme catalysis and the CUMPCD to $<1 \%$ of controls $(3,17$, 19). Previously, we found that, of nine women studied, all seven with $<5 \%$ CUMPCD had premature ovarian failure, whereas those with $>5 \%$ galactose oxidation had normal ovarian function (20).

In this prospective study, we validated a simplified test of total body ${ }^{13} \mathrm{C}$-D-galactose oxidation from an oral bolus to ${ }^{13} \mathrm{CO}_{2}$ in expired air (the "breath test") against mutant hGALT alleles of known severity. We then compared this breath test against four other biochemical parameters as potential risk indicators for verbal dyspraxia. We eliminated erythrocyte hGALT activity and highest galactose-1-phosphate concentrations as potential risk indicators and quantified the odds for developing dyspraxia associated with the breath test, mean erythrocyte galactose-1-phosphate concentrations, and mean urinary galactitol excretion.

\section{METHODS}

Study design and subjects. Fifty-five individuals participated in the evaluation of a simplified, noninvasive breath test. Subjects included 13 normal controls and 42 patients with galactosemia. Data on these subjects were used to quantify and standardize a simplified breath test at five time points: baseline and $90,100,110$, and $120 \mathrm{~min}$.

We used a case-control design to analyze the outcome, dyspraxia. Criteria for entry into the dyspraxia study included erythrocyte hGALT activity $<3 \%$, older than 2.5 years, completion of the breath test, and consent to a speech evaluation. Seven of the 42 patients were too young and as such were ineligible for the dyspraxia study. Of the remaining 35 , eight patients were lost to follow-up and three refused. Twenty-four patients satisfied the eligibility criteria and consented to formal speech evaluation by a licensed speech therapist. Completed formal speech evaluations were obtained on all 24 patients. All protocols were approved by the Emory Institutional Review Board, and informed consent was obtained from each volunteer.

Determining total body oxidation of ${ }^{13} C$-D-galactose to ${ }^{13} \mathrm{CO}_{2}$ (breath test). After a baseline breath collection, patients and control volunteers were administered an oral bolus of $7 \mathrm{mg}$ of $1-C^{13}$-D-galactose per kilogram of body weight and breath was collected at $90,100,110$, and $120 \mathrm{~min} .{ }^{13} \mathrm{C}$ enrichment of
$\mathrm{CO}_{2}$ was measured in collected breath samples by automated, gas-isotope ratio, mass spectrometry as previously described $(16,17,21)$. Micromoles of expired ${ }^{13} \mathrm{CO}_{2}$ per minute were determined from the estimated $\mathrm{CO}_{2}$ production rate obtained from calculations using the Weir equation (22) based on a fasting respiratory quotient (RQ) of 0.80 . From the estimated $\mathrm{CO}_{2}$ production rate, the basal metabolic rate was obtained by the Schofield equation (23). Using the calculated $\mathrm{CO}_{2}$ production rate and the isotopic enrichment values, PCD recovered was calculated at each time point and a curve of PCD recovered was generated (21). A CUMPCD recovered for each time period was then derived by integrating the area under the PCD curve using the trapezoidal method. The impact of the oral bolus of D-galactose in patients with galactosemia was determined by quantifying erythrocyte galactose-1-phosphate and urinary galactitol concentrations before and after D-galactose administration.

Apraxia Profile. Licensed speech pathologists administered the Apraxia Profile to 21 patients (24). The Apraxia Profile tests the ability of the patient to complete numerous verbal tasks and includes an oral motor exam, a diadochokinesis test, word imitation, testing prosody with phrases and sentences, and a connected speech sample test that assesses volitional speech intelligibility (24). Three patients were evaluated for dyspraxia by licensed speech pathologists through the Atlanta Area School System. A discontinuous assignment of dyspraxia present (affected) or absent (unaffected) was made. Two patients, classified as "affected," had dyspraxia in early childhood. They were treated with $6-8$ y of intensive speech therapy and subsequently had normal Apraxia Profiles at the time of this study.

Biochemical phenotyping and molecular genotyping. Biochemical phenotyping and molecular genotyping of hGALT were performed using previously described methods $(5,25)$. Highest galactose-1-phosphate was defined as the highest reported concentration of galactose-1-phosphate in the peripheral blood cells and served as a proxy for clinical severity during the neonatal period. Mean erythrocyte galactose-1-phosphate was the average of the five most recent values obtained after therapeutic levels were achieved through dietary galactose restriction. Urinary galactitol concentrations were quantified using gas chromatography-mass spectrometry as previously described (9). Mean urinary galactitol was defined as the average of the five most recent urinary galactitol concentrations measured simultaneously with other "risk factors."

Data analysis. Mean CUMPCD values were compared among the dyspraxic and nondyspraxic groups using the $t$ test. Reproducibility and stability over age were estimated using multiple breath tests on four normal subjects. We analyzed reproducibility with a nested, random effects analysis of variance. For evaluating the effect of age on the test, Spearman's correlation coefficients were calculated for percentage change in age and its relationship with percentage change in CUMPCD values at $120 \mathrm{~min}$. Paired $t$ tests assessed the impact of the oral bolus on the metabolite levels of patients with galactosemia. Significance among the means of the biochemical variables was determined with respect to the presence or absence of dyspraxic speech by Wilcoxon rank sum or ANOVA. Contin- 
uous biochemical variables were correlated using Pearson's correlations coefficients.

Exposure variables that differed significantly between patients with dyspraxic speech and patients with normal speech were evaluated for potential risk. The SAS SENSIT program was used to obtain sensitivities and specificities for the exposure variables. The cutoff point achieving the best balance of sensitivity and specificity as indicated by the highest correct score was used in regression modeling for risk. Logistic regression was used to obtain unadjusted and adjusted odds ratios (OR) and associated $95 \%$ confidence intervals for the exposure variables. Adjusted OR were obtained using a logistic regression model in which the outcome variable, verbal dyspraxia, was modeled separately against the exposure variable. We controlled for the following confounders: erythrocyte GALT activity, race, sex, and age at speech evaluation. GALT activity and age were continuous variables, whereas race and sex were discontinuous. Race was categorized as either white or other. All statistical analyses were performed using Microsoft Excel and SAS statistical software version 8.0 (SAS Institute Inc, Cary, NC, U.S.A.); $p<0.05$ was considered significant (26).

\section{RESULTS}

The CUMPD of ${ }^{13} \mathrm{C}$-D-galactose recovered as ${ }^{13} \mathrm{CO}_{2}$ was plotted for all 42 patients with galactosemia (Fig. 1). An empiric cutoff that maximally discriminates for the presence of dyspraxia is evident at 5\% CUMPCD. We had previously found a similar discriminate for CUMCPD in validating a more complex breath test against genotype (20). Of the 24 patients who participated in the outcomes analysis for verbal dyspraxia, 13 had dyspraxia and fell below the 5\% cutoff. Thus, we continued to stratify patients as $<5 \%$ CUMPCD or $\geq 5 \%$ CUMPCD on the basis of this empiric observation in subsequent outcomes analysis.

Twenty-eight of the 42 patients with galactosemia had CUMPCD values of $<5 \%$ at 120 min after bolus administra-

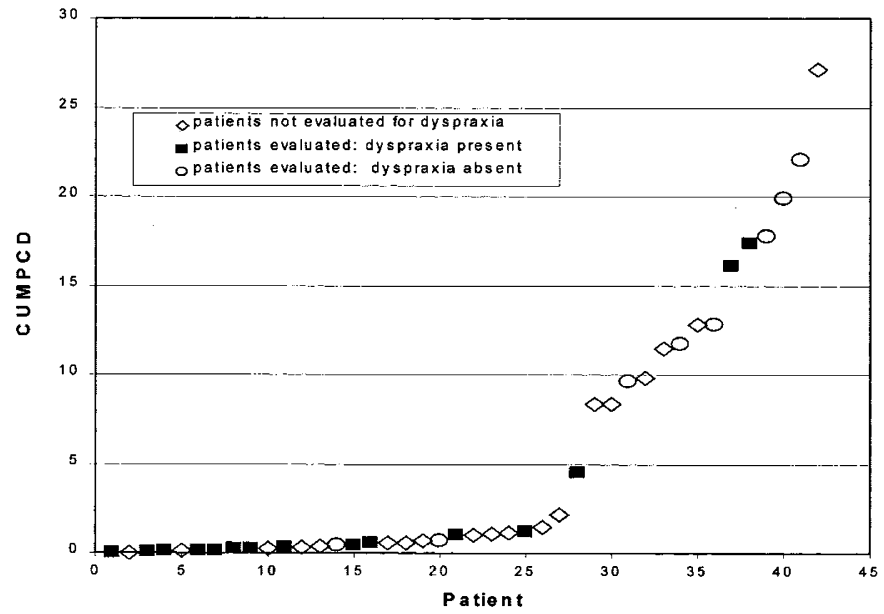

Figure 1. Scatterplot for cumulative percent dose of ${ }^{13} \mathrm{D}$-[D]-galactose recovered as ${ }^{13} \mathrm{CO}_{2}$ in breath at 120 minutes from 42 patients with galactosemia (CUMPCD). All symbols represent results of breath testing in all patients: $\diamond$, patients who did not participate in the dyspraxia study; $\mathbf{\square}$, patients with dyspraxia; $\bigcirc$, those without dyspraxia. tion. Mutations that produced this degree of impairment in galactose oxidation included Q188R, 5-kb deletion, K285N, Y209C, V151A, L358X, L195P, E308 K, R204X, and IVS-F. All of these mutations produce severe impairment of the hGALT enzyme $(3,27)$. In contrast, "leaky" mutations that impaired total body galactose oxidation to a lesser degree included S135L, F171S, D113N, E203 K in cis with N314D, T138M, S293F, D98N, and H321Y (Table 1). These leaky mutations may produce absent erythrocyte hGALT but have residual in vivo or in vitro activity $(3,5,18,19,27)$.

Patients with CUMPCD values $<5 \%$ at $120 \mathrm{~min}$ also had significantly less galactose oxidation to $\mathrm{CO}_{2}$ at 90, 100, and 110 min than controls. Normal individuals and patients with CUMPCD values $>5 \%$ did not differ significantly from one another at any time point (data not shown).

Four normal adults underwent multiple breath tests to estimate the inter- and intraperson variability of the test and the stability of the test results with increasing age. Two normal subjects had two tests each with $1.5 \mathrm{y}$ elapsing between administration of the first and the second tests. The other two normal subjects underwent three rounds of testing, with each test separated by 1-2 mo. The overall variance for the breath test was \pm 4.08 with $19.8 \%$ of the variance as a result of intraperson variability. This sample, composed only of adults, was insufficient for statistical analysis of an age effect on breath test results.

We assessed the impact of the oral bolus on metabolite levels and observed small increases from baseline to 120 min for erythrocyte galactose-1-phosphate and urinary galactitol concentrations. These increases were not considered clinically significant and promptly reverted to baseline over $12 \mathrm{~h}$ (data not shown).

Twenty-four patients with galactosemia participated in the outcomes analysis for verbal dyspraxia. Their demographic and biochemical parameters were generalized in Table 2. Of these patients, 15 were dyspraxic. Seventy percent were white, and roughly half were male. Mean CUMPCD was $6.10 \pm$ $7.69 \%$. Fifteen of the 24 patients had values $<5 \%$. Average mean erythrocyte hGALT activity was $0.33 \pm 0.69$ pmol of UDP ${ }^{14} \mathrm{C}-\mathrm{Gal} / \mathrm{g} \mathrm{Hbg}{ }^{-1} / \mathrm{min}^{-1}$. Average highest erythrocyte galactose-1-phosphate was $63.8 \pm 72.3 \mathrm{mg} / \mathrm{dL}$. Data for highest galactose-1-phosphate was not available for five patients. Average erythrocyte galactose-1-phosphate concentration was $2.83 \pm 1.28 \mathrm{mg} / \mathrm{dL}$, and average mean urinary galactitol was $166 \pm 76.2 \mathrm{mmol} / \mathrm{mol}$ creatinine.

We characterized these biochemical parameters among the 24 patients with galactosemia with regard to the presence or absence of dyspraxia (Table 3 ). Of the 15 patients with dyspraxia, the average CUMPCD value was $2.84 \pm 5.76 \%$. This value was significantly lower than the CUMPCD of patients without dyspraxia (CUMPCD $=11.51 \pm 7.67 \% ; p=0.008$ ). Both mean erythrocyte galactose-1-phosphate concentrations and mean urinary galactitol excretion were significantly higher among patients with dyspraxia $(p<0.05)$. However, patients with dyspraxia did not differ significantly in GALT activity or highest galactose-1-phosphate concentration from patients with normal speech.

We evaluated the relationship among the five potential biochemical parameters that might affect dyspraxic outcome. Highest galactose-1-phosphate and erythrocyte hGALT activ- 
Table 1. Genotype distribution of all patients participating in the breath test and the subset and who also underwent analysis for verbal dyspraxia

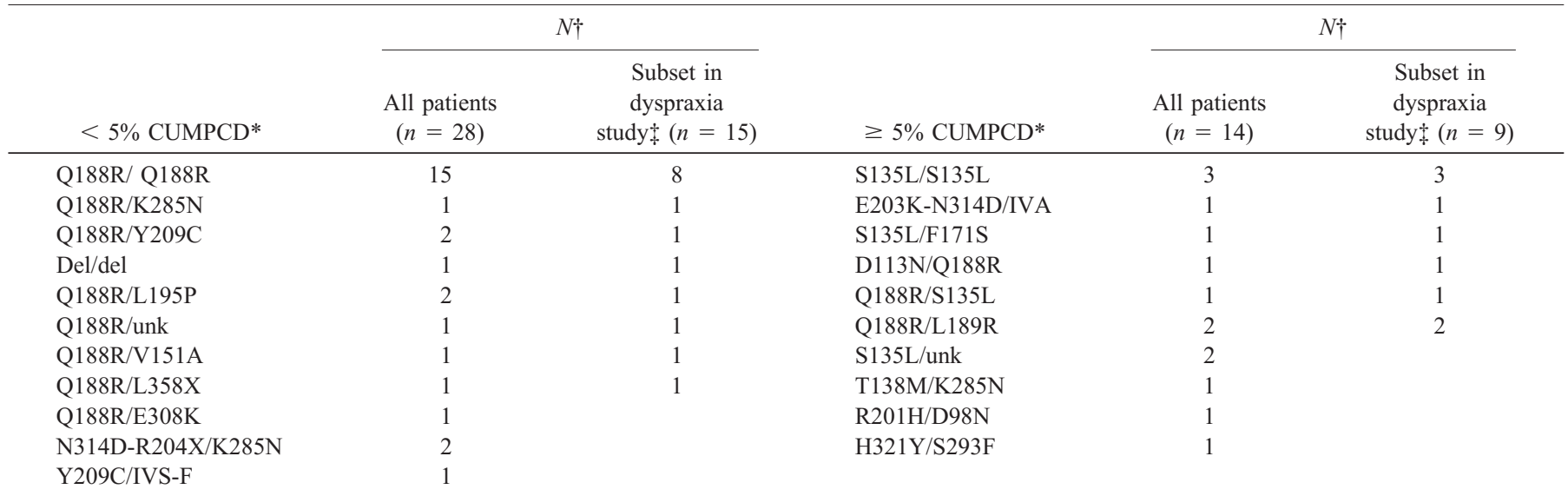

* Stratification by CUMPCD values less than or greater than 5\% was determined by scatterplot analysis of CUMPCD values at 120 min as a discriminate analysis for dyspraxia

$\dagger$ Numbers of subjects for each genotype and category are given.

$\ddagger$ Represent the subset of patients who participated in the breath test study.

Table 2. General characteristics among patients with galactosemia in the dyspraxia study population

\begin{tabular}{|c|c|c|}
\hline Variable & Frequency or mean & $N$ \\
\hline White & $17(70.8 \%)^{*}$ & 24 \\
\hline Male & $11(45.8 \%)$ & 24 \\
\hline Dyspraxic & $15(62.5 \%)$ & 24 \\
\hline Age at speech evaluation (y) & $9 \mathrm{yr} 2 \mathrm{~m} \pm 6 \mathrm{y} 2 \mathrm{~m}(2.5-30) \dagger$ & 24 \\
\hline Age at breath test $(\mathrm{y})$ & $8 \mathrm{y} 10 \mathrm{~m} \pm 5 \mathrm{y} 6 \mathrm{~m}(2.3-29)$ & 24 \\
\hline $\begin{array}{l}\text { Erythrocyte hGALT activity } \\
\quad\left(\text { pmols UDP }-{ }^{14} \mathrm{C} \text { Gal } \cdot \mathrm{g} \mathrm{Hbg} \cdot \mathrm{min}\right)\end{array}$ & $0.330 \pm 0.690(0-2.8)$ & 24 \\
\hline Mean galactose-1-phosphate (mg/dL) & $2.83 \pm 1.28(0.162-4.78)$ & 24 \\
\hline Highest galactose-1-phosphate (mg/dL) & $63.8 \pm 72.3(4.32-272) \div$ & 19 \\
\hline Mean urinary galactitol (mmol/mol creatinine) & $166 \pm 76.2(38.2-335)$ & 24 \\
\hline
\end{tabular}

* Discontinous values are expressed as the proportion with the percentage in parentheses.

$\dagger$ Continuous variables are expressed as the mean $\pm \mathrm{SD}$ with ranges given in parentheses.

\$ Data for highest galactose-1-phosphate was not available for five individuals.

Table 3. Significance of independent biochemical variables to dyspraxic speech

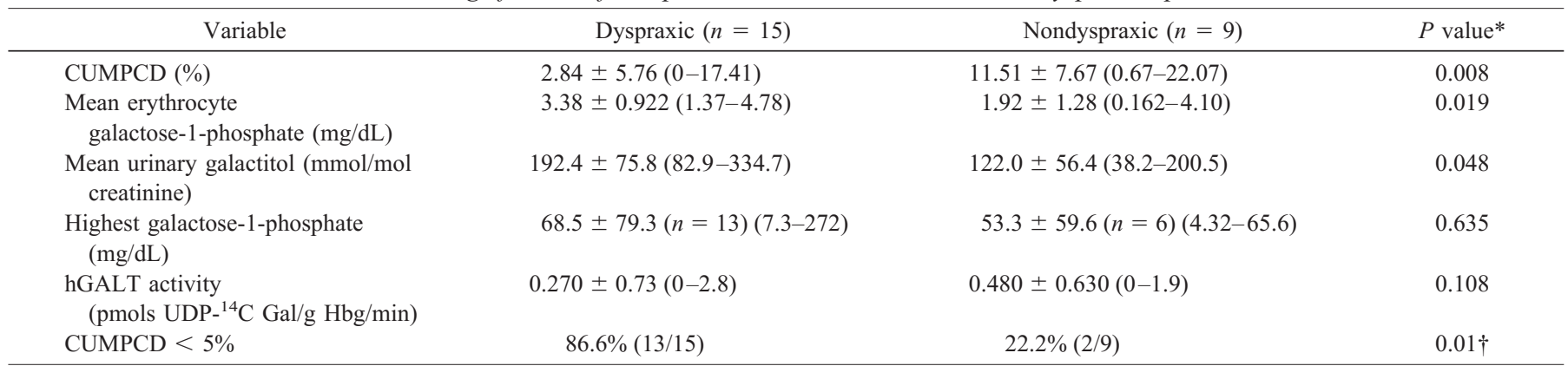

* $P$ values for continuous variables were determined by Wilcoxon rank sum. Values for continuous variables are expressed as the mean $\pm \mathrm{SD}$, and ranges are given in parentheses.

$\dagger P$ values for noncontinuous variables were determined by ANOVA. Values for noncontinuous variables are expressed as a percentage with the proportion in parentheses

ity did not correlate with CUMPCD $(r=-0.017, p=0.945$; $r=0.063, p=0.768)$, mean erythrocyte galactose-1-phosphate $(r=0.087, p=0.724 ; r=0.030, p=0.889)$, or mean urinary galactitol $(r=-0.068, p=0.783 ; r=0.150$, $p=0.482)$. CUMPCD correlated inversely with mean erythrocyte galactose-1-phosphate $(r=-0.863, p<0.001)$ and mean urinary galactitol $(r=-0.540, p<0.007)$. A significant, although weak, correlation existed between mean urinary galactitol and mean erythrocyte galactose-1-phosphate $(r=0.560, p<0.005$; data not shown). On the basis of the lack of significance and correlation for highest galactose-1-phosphate and hGALT activity, we eliminated these variables from further risk analysis.

We investigated the best exposure values for CUMPCD, mean galactose-1-phosphate, and mean urinary galactitol for 
predicting the presence or absence of verbal dyspraxia (Table 4). For CUMPCD, 5\% was chosen as the cutoff point for predicting dyspraxia as this value achieved the greatest balance of sensitivity and specificity as indicated by the highest correct score $($ sensitivity $=86.7$, specificity $=77.8$, correct score $=$ 83.3). For mean erythrocyte galactose-1-phosphate concentrations, the greatest balance for sensitivity and specificity for predicting dyspraxia was observed at the $2.7 \mathrm{mg} / \mathrm{dL}$ cutoff level (sensitivity $=84.6$, specificity $=66.7$, correct score $=79.2$ ). This value is lower than a previously used level of $3.28 \mathrm{mg} / \mathrm{dL}$ (10). In this study, we found the $3.28 \mathrm{mg} / \mathrm{dL}$ cutoff to have a lower sensitivity (40\%) and decreased correct score (54.2) for dyspraxic outcome, although specificity did increase slightly to 77.8 (data not shown). Interestingly, all patients with CUMPCD values $<5 \%$ had mean galactose-1-phosphate concentrations $>2.7 \mathrm{mg} / \mathrm{dL}$. A cutoff for urinary galactitol at 135 $\mathrm{mmol} / \mathrm{mol}$ creatinine level achieved the highest overall correct score for this variable (correct score $=70.8$ sensitivity $=80.8$, specificity $=55.6$ ) and was used as the cutoff point in risk assessment.

As seen in Table 4, CUMPCD values $<5 \%$ were strongly and significantly associated with risk for dyspraxic outcome after controlling for confounders [adjusted OR $=21.1$ (1.68, $265)]$. We observed a strong association with dyspraxic outcome for both mean galactose-1-phosphate levels $>2.7 \mathrm{mg} / \mathrm{dL}$ and urinary galactitol $>135 \mathrm{mmol} / \mathrm{mol}$ creatinine. However, only mean galactose-1-phosphate was significant after controlling for confounders. These data indicate that the breath test was the most sensitive, specific, and strongly associated predictor of verbal dyspraxia among this galactosemic population.

\section{DISCUSSION}

Here we confirm that, using only four time points, a simplified 2-h breath test is a reproducible and noninvasive method by which to measure total body oxidation of galactose in patients with galactosemia. Patients with total body galactose oxidation of $<5 \%$ included individuals with two mutant alleles known to impair all hGALT activity. These included Q188R, $\mathrm{K} 285 \mathrm{~N}$, and the $5-\mathrm{kb}$ deletion seen in the Ashkenazi population $(3,27)$. Conversely, when "variant" $G$ alleles were present, the CUMPCD was $\geq 5 \%$. These variant mutations included the
S135L and T138M alleles, which alter hGALT stability without ablating the catalytic site of the protein $(3,18,19)$.

The breath test was the best predictor of dyspraxia among the five biochemical parameters compared (Tables 3 and 4). Highest erythrocyte galactose-1-phosphate concentrations and erythrocyte GALT activity neither correlated with other biochemical parameters nor were significantly different between patients with dyspraxia and those with normal speech. By comparison, the CUMPCD, mean erythrocyte galactose-1phosphate, and mean urinary galactitol all predicted dyspraxic outcome, with CUMPCD having the greatest overall sensitivity, specificity, and associated risk.

Using the Apraxia Profile, we found dyspraxia to have a prevalence of $\sim 65 \%$ among this population of patients with galactosemia. Bias toward individuals with dyspraxia is a problem in a study such as this one, as patients with speech problems may be more willing to undergo testing. However, the prevalence of dyspraxia in our study was consistent with the prevalence observed in other studies; thus, we do not believe that selection bias was a significant issue $(10,14)$. A search for early biochemical predictors is significant to dyspraxia in that early detection of this speech problem provides a basis for early intervention and treatment (28). Diagnosis and further evaluation of children with galactosemia before the development of volitional speech will aid physicians and parents in assessing a child's risk for dyspraxia and thus enable proper interventions. Studies by Waggoner et al. (7) indicated that the diminished IQ and DQ scores seen in some patients with classical galactosemia were associated with verbal ability. Therefore, preventing poor academic performance with proper interventions for dyspraxia at an early age will allow patients to participate in normal mental and behavioral development. Two patients in this study supported this notion. They had significantly impaired speech at ages $2-3$ y but returned normal Apraxia Profiles as adolescents after years of intensive speech therapy. Both had CUMPCD values $<5 \%$.

Elevated mean erythrocyte galactose-1-phosphate levels are associated with dyspraxic outcome (10). In this study, we found that values exceeding $2.7 \mathrm{mg} / \mathrm{dL}$ were strongly associated with dyspraxic outcome, and this association was significant despite a small and highly variable sample size (Table 4).

Table 4. Predictive values for dyspraxia among significant biochemical parameters in galactosemia

\begin{tabular}{|c|c|c|c|c|c|c|c|}
\hline & $\begin{array}{l}\text { Unadjusted OR } \\
\text { (CI)* }\end{array}$ & $\begin{array}{l}\text { Adjusted OR } \\
(\mathrm{CI}) \dagger\end{array}$ & Sensitivity & Specificity & $\begin{array}{l}\text { Correct } \\
\text { score }\end{array}$ & PPV & NPV \\
\hline CUMPCD $<5 \%$ & $22.7(2.6-198)$ & $21.1(1.68-265)$ & 86.7 & 77.8 & 83.3 & 87 & 78 \\
\hline $\begin{array}{l}\text { Mean erythrocyte } \\
\text { galactose-1- } \\
\text { phosphate }>2.7 \\
\text { mg/dL }\end{array}$ & $13.01(1.71-99.4)$ & $12.83(1.81-139)$ & 86.7 & 86.7 & 79.2 & 87 & 78 \\
\hline $\begin{array}{l}\text { Mean urinary galactitol } \\
>135 \mathrm{mmol} / \mathrm{mol} \\
\text { creatinine }\end{array}$ & $5.0(0.81-36.1)$ & $4.58(0.57-36.6)$ & 80.8 & 55.6 & 70.8 & 73 & 73 \\
\hline
\end{tabular}

CI, confidence interval; PPV, positive predictive value; NPV, negtive predictive value.

* Unadjusted OR and CI were obtained by logistic regression in which dyspraxia as a yes or no outcome was modeled against the variable of interest which was coded as exposed or unexposed.

$\dagger$ Adjusted OR and CI were calculated using a logistic regression model controlling for hGALT activity, race, sex, and age at speech evaluation.

$\ddagger$ Sensitivities, specificities, correct scores, NPV, and PPV were calculated using SASSensit version 8.0. 
The association of increased intracellular galactose-1phosphate with dyspraxic outcome may have a biochemical mechanism because galactose-1-phosphate competitively inhibits UDP-glucose pyrophosphorylase (hUGP2) (29, 30). Thus, intracellular accumulation of galactose-1-phosphate may reduce intracellular UDP-galactose (UDP-gal) and UDPglucose (UDP-glu) or alter their ratios, and impair synthesis of glycolipids, glycoproteins, and glycogen.

Galactitol accumulation in lens fibers is associated with cataract formation in human galactokinase and hGALT deficiencies $(2,31,32)$. Galactitol is produced from excess Dgalactose by aldose reductase, when intracellular concentrations of D-galactose exceed the capacity of hGALT or human galactokinase (33). Because galactitol is not further metabolized, it accumulates in cells, produces osmotic effects that alter selective cell permeability, and consequently promotes cell death. Age- and treatment-dependent ranges for urinary galactitol excretion in patients with galactosemia have been determined (34). Patients with poor dietary control excrete higher concentrations of urinary galactitol than compliant galactosemics $(8000-69,000 \mathrm{mmol} / \mathrm{mol}$ creatinine versus $45-900$ $\mathrm{mmol} / \mathrm{mol}$ creatinine). The range for compliant galactosemics, however, still exceeds that of normal controls $(96-170 \mathrm{mmol} /$ mol creatinine). In this study, we established that urinary galactitol excretion exceeding $135 \mathrm{mmol} / \mathrm{mol}$ creatinine was a strong though nonsignificant biologic parameter associated with dyspraxic outcome. It should be noted that this risk indicator was not as strongly associated with dyspraxic outcome as CUMPCD values $<5 \%$ or mean erythrocyte galactose-1-phosphate concentrations $>2.7 \mathrm{mg} / \mathrm{dL}$ (Table 4).

One clinical conundrum is that galactokinase deficiency produces increased galactitol production and cataract formation but not hepatotoxicity or neurologic impairments (2). However, there are many hypothetical mechanisms by which elevated galactitol, in association with other insulting analytes, could be a neurotoxin (35-39). Galactitol reduced the antioxidant potential of cells and the glutathione redox potential (35, 36). Galactitol accumulated in the brain of children with galactosemia and was associated with neurotoxicity (36-39). Therefore, galactitol accumulation alone may not cause brain damage, as evidenced by galactokinase deficiency. However, in association with hGALT deficiency, galactose-1-phosphate accumulation, and altered UDP-gal/UDP-glu ratios, elevated galactitol is an important factor that increases the probability of developing dyspraxia (Table 4).

Aberrant glycosylation and galactosylation of macromolecules, including complex carbohydrates (40), folliclestimulating hormone and follicle-stimulating hormone receptors (41), serum transferrin (42), and brain glycolipids (43), have been observed in patients with classical galactosemia and implicated in the long-term reproductive and neurologic sequelae. Proper posttranslational processing of glycolipids and glycoproteins requires regulated concentrations of UDPhexoses (44). Several studies have demonstrated that patients with classical galactosemia have significantly altered concentrations and ratios of UDP-gal and UDP-glu in erythrocytes $(45,46)$. Although quantification of these compounds was beyond the capabilities of this study, it would be interesting to assess UDP-gal and UDP-glu and correlate these analytes with CUMPCD and mean erythrocyte galactose-1-phosphate. One could postulate that individuals with less severe impairment of hGALT activity and, thus, residual, hepatic hGALT activity could metabolize some galactose-1-phosphate, reduce galactose-1-phosphate concentrations, decrease the inhibitory effects on hUGP2, and enable continued production of UDP-glu. hUGP2 and epimerase become critical enzymes in hGALT deficiency as they are required to maintain UDP-glu and UDP-gal levels when hGALT cannot produce adequate amounts of UDP-gal (2).

Understanding galactose and uridylhexose homeostasis in biologic systems remains a fundamental challenge. In the interim, in patients with galactosemia, total body galactose oxidation, mean erythrocyte galactose-1-phosphate, and mean urinary galactitol are associated with the development of dyspraxic speech. Further research into the biochemical mechanisms underlying the development of normal and abnormal speech is needed to elucidate the contributing role of these factors to this outcome.

Aknowledgments. We thank Dr. Amita Manatunga, George Cotsonis, and Azhar Nizam for statistical guidance and advice. We thank the Emory Genetics Lab, Dr. Phillip Dembure, and Sharon Langley for biochemical phenotyping and molecular genotyping of analytes and GALT. We thank Dr. Stan Konopka of ABD, LLC, for quantifying ${ }^{13} \mathrm{CO}_{2}$ in breath. We are also thankful for the participation of the patients and their referring physicians, without whom this research would not be possible. We thank Drs. Paul Fernhoff and R. Dwain Blackston for aiding in the management of the patients.

\section{REFERENCES}

1. Beutler E, Beluda ML, Sturgeon P, Day RW 1995 A new genetic abnormality resulting in galactose-1-phosphate deficiency. Lancet 1:353-355

2. Holton JB, Walter JH, Tyfield LA 2001 Galactosemia. In: Scriver CR, Beaudet AL, Sly WS, Valle D (eds) The Metabolic and Molecular Basis of Inherited Disease. McGraw-Hill, New York, pp 1553-1587

3. Elsas LJ, Lai K 1998 The molecular biology of galactosemia. Genet Med 1:40-48. Available at: www.pediatrics/medgen/research/db.html

4. Tyfield L, Reichardt J, Fridovich-Keil J, Croke DT, Elsas LJ, Strobl W, Kozak L, Coskun T, Novelli G, Okano Y, Zekanowski C, Shin Y, Boleda MD 1999 Classical galactosemia and mutations at the galactose-1-phosphate uridyl transferase (GALT) gene. Hum Mutat 13:417-430. Available at: www.ich.bris.ac.uk/galtda/ mutations.html

5. Elsas LJ, Langley SD, Steele R, Eringer J, Fridovich-Keil JL, Brown A, Singh R, Fernhoff P, Hjelm LN, Dembure PP 1995 Galactosemia: a strategy to identify new biochemical phenotypes and molecular genotypes. Am J Hum Gen 56:630-639

6. Elsas LJ, Fernhoff P, Brown A 1983 Newborn screening for metabolic disorders in the state of Georgia. In: Naruse H, Irie M (eds) Neonatal Screening: Proceedings of the International Symposium on Neonatal Screening for Inborn Errors of Metabolism. Excerpta Medica, Tokyo, pp 117-118

7. Waggoner DD, Buist NRM, Donnell GN 1990 Long-term prognosis in galactosemia: results of a survey of 350 cases. J Inherit Metab Dis 13:802-818

8. Berry GT, Nissim I, Lin Z, Mazur A, Gibson JB, Segal S 1995 Endogenous synthesis of galactose in normal men and patients with hereditary galactosemia. Lancet 346:1073-1074

9. Berry GT, Palmieri M, Gross KC, Acosta PB, Henstenberg JA, Mazur A, Reynolds R, Segal S 1993 The effects of dietary fruits and vegetables on urinary galactitol excretion in galactose-1-phosphate uridyltransferase deficiency. J Inherit Metab Dis 16:91-100

10. Robertson A, Singh RH, Guerrero NV, Hundley M, Elsas LJ 2000 Outcomes analysis of verbal dyspraxia in classic galactosemia. Genet Med 2:142-148

11. Schweitzer S, Shin Y, Jakobs C, Brodehl J 1993 Long term outcome in 134 patients with galactosemia. Eur J Pediatr 154:S45-S49

12. Hansen TWR, Henrichsen B, Rasmussen RK, Carling A, Andreassen AB Skjeldal O 1996 Neuropsychological and linguistic follow-up studies of children with galactosaemia from an unscreened population. Acta Pediatr 85:1197-1201 
13. Kaufman FR, Reichardt JKV, Ng WG, Xu YK, Manis FR, McBride-Chang C, Wolff JA 1994 Correlation of cognitive, neurologic, and ovarian outcome with the Q188R mutation of the galactose-1-phosphate uridyltransferase gene. J Pediatr 125:225-227

14. Nelson DC 1995 Verbal dyspraxia in children with galactosemia. Eur J Pediatr 154(S2):S6-S7

15. Nelson DC, Waggoner DD, Donnell GD, Tuerck JM, Buist NRM 1991 Verbal dyspraxia in treated galactosemia. Pediatrics 88:346-350

16. Berry GT, Nissim I, Gibson JB, Mazur AT, Lin Z, Elsas LJ, Singh RH, Klein PD, Segal S 1997 Quantitative assessment of whole body galactose metabolism in galactosemic patients. Eur J Pediatr 156(S1):S43-S49

17. Berry GT, Singh RH, Mazur AT, Palmieri MJ, Guerrero NV, Kennedy MJ, Chen J, Reynolds R, Palmieri MJ, Klein PD, Segal S, Elsas LJE 2000 Galactose breath testing distinguishes variant and severe galactose-1-phosphate uridyltransferase genotypes. J Pediatr Res 48:323-328

18. Lai K, Langley SD, Singh RS, Dembure PP, Hjelm LN, Elsas LJ 1996 A prevalent mutation for galactosemia among black Americans. J Pediatr 128:89-95

19. Lai K, Elsas LJ 2001 Structure-function analysis of a common mutation in blacks with transferase-deficiency galactosemia. Mol Genet Metab 74:264-272

20. Guerrero NV, Singh RH, Manatunga A, Berry GT, Steiner RD, Elsas LJ 2000 Risk factors for premature ovarian failure in females with galactosemia. J Pediatr 137:833841

21. Elsas LJ, Ellerine NP, Klein PD 1993 Practical methods to estimate whole body leucine oxidation in maple syrup urine disease. Pediatr Res 33:445-451

22. de Weir JR 1949 New methods for calculating metabolic rate with special reference to protein metabolism. J Physiol 109:1-9

23. Schofield WN 1985 Predicting basal metabolic rate, new standards and review of previous work. Hum Nutr Clin Nutr 39C:5-41

24. Hickman LA 1997 The Apraxia Profile. A Descriptive Assessment Tool. Communication Skill Builders, a division of the Psychological Corporation, San Antonio, Texas

25. Bernmeyer H 1974 Galactose-1-P: determination as galactose after hydrolysis by alkaline phosphatase. In: Bermeyer H (ed) Methods of Enzymatic Analysis. pp 1291-1295. Verlag Chemie International, Deerfield Beach, FL

26. Rosner B 1994 Fundamentals of Biostatistics, 4th Ed. Wadsworth Publishing Co. New York

27. Lai K, Willis A, Elsas LJ 1999 The biochemical role of glutamine 188 in human galactose-1-phosphate uridyltransferase. J Biol Chem 274:6559-6566

28. Hodge MN, Hancock HR 1994 Assessment of children with developmental apraxia of speech: a rationale. Clin Commun Disord 4:91-101

29. Gitzelmann R 1995 Galactose-1-phosphate in the pathophysiology of galactosemia. Eur J Pediatr 154:S45-S49

30. Lai K, Elsas LJ 2000 Overexpression of human UDP-glucose pyrophosphorylase rescues galactose-1-phosphate uridyltransferase deficient yeast. Biochem Biophys Res Commun 271:392-400

31. Stambolian D 1988 Galactose and cataract. Surv Ophthalmol 32:333-349
32. Ai Y, Zheng Z, O'Brian-Jenkins A, Bernard DJ, Wynshaw-Boris T, Ning C, Reynolds R, Segal S, Huang K, Stambolian D 2000 A mouse model of galactose-induced cataracts. Hum Mol Genet 9:1821-1827

33. Berry GT 1995 The role of polyols in the pathophysiology of hypergalactosemia. Eur J Pediatr 154:S53-S64

34. Jacobs C, Schweitzer S, Dorland B 1995 Galactitol in galactosemia. Eur J Pediatr 154:S45-S49

35. Green DA, Lang HJ, Stevens MJ, Obrosova IG 1999 Evaluation of aldose reductase inhibitor vs. sorbitol dehydrogenase inhibitor on diabetes-induced changes in retinal $\mathrm{NAD}(\mathrm{P})$-redox status: support for the role of aldose reductase but not sorbitol dehydrogenase in diabetic retinopathy. Invest Ophthalmol Vis Sci 40:S370

36. Kashiwagi A, Asahani T, Ikebuchi M, Tanaka Y, Takagi Y, Nishio Y, Kikkawa R, Shigeta Y 1994 Abnormal glutathione metabolism and increased cytotoxicity of caused $\mathrm{H}_{2} \mathrm{O}_{2}$ in human umbilical vein endothelial cells cultured in high glucose medium. Diabetologia 37:264-269

37. Qaun MR, Wells HJ, Wells WW, Sherman FE, Egan TJ 1966 Galactitol in the tissues of a galactosemic child. Am J Dis Child 112:477-478

38. Berry GT, Hunter JV, Wang Z, Dreha S, Mazur A, Brooks DG, Ning C, Zimmerman RA, Segal S 2001 In vivo evidence of brain galactitol accumulation in an infant with galactosemia and encephalopathy. J Pediatr 138:260-262

39. Wang ZJ, Berry GT, Dreha SF, Zhao H, Segal S, Zimmerman RA 2001 Proton magnetic resonance spectroscopy of brain metabolites in galactosemia. Ann Neurol 50:266-269

40. Ornstein KS, McGuire J, Berry GT, Roth S, Segal S 1992 Abnormal galactosylation of complex carbohydrates in cultured fibroblasts from patients with galactose-1phosphate uridyltransferase deficiency. Pediatr Res 31:508-511

41. Prestoz LLC, Couto AS, Shin YS, Petry G 1997 Altered follicle stimulating hormone isoforms in female galactosaemia patients. Eur J Pediatr 156:116-120

42. Charlewood J, Clayton P, Keir G, Mian N, Winchester B 1998 Defective galactosylation of serum transferrin in galactosemia. Glycobiology 8:351-357

43. Petry K, Greinix HT, Nudelman E, Eisen H, Hakomori SI, Levy H, Reichardt JKV 1991 Characterization of a novel biochemical abnormality in galactosemia: deficiency of glycolipids containing galactose or $\mathrm{N}$-acetylgalactosamine and accumulation of precursors in brain and lymphocytes. Biochem Med Metab Biol 46:93-104

44. Hakomori S 1990 Bifunctional role of glycosphingolipids. J Biol Chem 265:1871318716

45. Ng WG, Xu YK, Kaufmann FR, Donnell GN 1989 Deficit of uridine diphosphate glucose and uridine diphosphate galactose in galactosemia. J Inherit Metab Dis 12:257-266

46. Gibson JB, Reynolds RA, Palmieri MJ, Berry GT, Elsas LJ, Levy H, Segal S 1995 Comparison of erythrocyte uridine sugar nucleotide levels in normals, classic galactosemics, and patients with other metabolic disorders. Metabolism 44:597-604 